\title{
Magnetic filter operation in hydrogen plasmas
}

\author{
St Kolev $^{1}$, St Lishev ${ }^{1}$, A Shivarova ${ }^{1}$, Kh Tarnev ${ }^{2}$ and R Wilhelm ${ }^{3}$ \\ ${ }^{1}$ Faculty of Physics, Sofia University, BG-1164 Sofia, Bulgaria \\ ${ }^{2}$ Department of Applied Physics, Technical University-Sofia, BG-1000 Sofia, Bulgaria \\ ${ }^{3}$ Max-Planck-Institut für Plasmaphysik, EURATOM Association, D-85740 Garching, \\ Germany \\ e-mail: ashiva@phys.uni-sofia.bg
}

\begin{abstract}
Fluid-plasma model description of the operation of a magnetic filter for electron cooling in gas-discharge plasmas is presented in the study. Directed to the use of weak magnetic fields in the sources of negative hydrogen ion beams for additional heating of fusion plasmas, hydrogen discharges have been considered. The numerical results obtained within a 2D model are stressed. The 1D model presented aims at showing main trends whereas the results obtained within the 3D model, also developed, come to confirm the 2D-model description. The models outline importance of the transport phenomena: electron-energy and charged-particle fluxes. Reduction of the thermal flux across the magnetic field together with thermal diffusion and diffusion, acting in a combination, are in the basis of the electron cooling and of the spatial distribution of the electron density. Effects due to the $(\mathbf{E} \times \mathbf{B})$-drift and to the diamagnetic drift form the fine spatial structure of the plasma-parameter variations.
\end{abstract}

PACS numbers: 52.25.Fi; 52.30.-q; 52.50.-b

\section{Introduction}

The development of sources of negative ion beams for fusion plasma heating by neutral beam injection [1-4] is one of the stimuli motivating the active research on low-pressure hydrogen discharges. In general, the sources of negative hydrogen ions with volume-production based processes as well as the hybrid sources where surface production is employed are tandem-type sources with a construction ensuring space separation of regions of high and low electron temperature [3-19]. Thus, electron cooling in the discharge is needed and this is provided by a magnetic filter.

The magnetic filter is a localized transverse magnetic field. Its effect for cooling the electrons has been proved both experimentally $[3,6,7,13,20-26]$, by probe diagnostics and measurements of the current density of the extracted negative ions, and theoretically $[6-12,15,27]$ by modelling and discussions on the mechanisms governing the operation of the filter. The fluid-plasma models $[6-10,15]$ of the magnetic filter involve importance of the transport processes. However, the different models stress different aspects as mechanisms of the filter operation: (i) reduction of the electron mobility and of the diffusion in magnetized plasmas acting in a combination with the temperature dependence of the Coulomb collision frequency, the latter considered as a factor ensuring lower diffusion of the hot electrons; (ii) thermal conductivity effects, again acting together with Coulomb collisions; (iii) importance of the Lorentz force showing evidence due to cancelled effects of the $(\mathbf{E} \times \mathbf{B})$ - and diamagnetic drifts; (iv) importance of the diamagnetic drift; (v) diffusion acting together with elastic electron-neutral collisions. Both 1D and 2D models have been developed, however, as it has been usually stressed, 2D models are needed for proper description of the problem. Due to the complexity of the description, simplifying assumptions, like neglecting of collisions for charge particle production and losses, involvement of parameters replacing 
variables, suppositions for a homogeneous magnetic field, for quasi-neutrality or for a given space variation of unknown quantities have been made. Obviously, when description of the total source of negative ion beams is aimed to be completed, such simplifying assumptions are unavoidable. Numerical simulations [12,18,27] based on particle-in-cell and Monte Carlo methods, also developed, stress mechanisms of the filter operation related to instabilities, turbulent diffusion and potential gaps accelerating the ions.

Although being motivated by the use of the magnetic filters in the sources of negative ion beams for fusion-plasma heating, this study is completely concentrated on the particular problem of the magnetic filter operation, i.e. it aims at describing the spatial redistribution of the plasma parameters in hydrogen discharges caused by an external magnetic field. The magnetic field is inhomogeneous, with a given Gaussian - profile along the length of the discharge vessel (a metal chamber). A power input with a given spatial distribution shapes the region driving the discharge production. The study presents results from a fluid plasma description of the magnetic filter operation within a $2 \mathrm{D}$ model. The two directions considered, respectively, along and across the discharge vessel, are both perpendicular to the magnetic field. Concerning the production and destruction of charged particles and neutral species, only main processes are taken into account. The discharge structure obtained is completed by the spatial distribution of electron temperature and density, concentrations of the three types of positive hydrogen ions $\left(\mathrm{H}^{+}, \mathrm{H}_{2}^{+}\right.$ and $\mathrm{H}_{3}^{+}$) and of the two types of neutral species (hydrogen atoms and molecules) and of the potential of the dc electric field in the discharge; negative hydrogen ions are not included in the description because with respect to the sources of negative ion beams - their significance is towards the extraction region of the source, behind the magnetic filter. Results for a zero magnetic field and for a finite (varying) magnetic field are compared. The 1D- and 3D-models also presented come, respectively, to show the main trends of the plasma behaviour and to confirm the description within the 2D model. The obtained results show that a reduction of the thermal flux in a transverse magnetic field and, respectively, an increased locality of the discharge maintenance when the electrons are magnetized are responsible for the electron cooling by the magnetic field. The thermal conductivity effects are so strong that the particular temperature dependence of a given type of collision frequency considered could not be of importance. This is confirmed by results for argon discharges, also shown here, which display the same trends of behaviour like in hydrogen discharges. Simultaneous action of diffusion and thermal diffusion across the magnetic field are responsible for the main trends of the spatial distribution of the charged particle densities. The $(\mathbf{E} \times \mathbf{B})$-drift and the diamagnetic drift as well as the thermal flux related to the diamagnetic drift are responsible for forming the final spatial structure of the plasma parameters.

\section{Formulation of the problem and basic set of equations}

Figure 1 presents schematically the configuration of the plasma volume and the location of the magnetic filter. The magnetic field of the filter, orientated along the $y$-axis $(\mathbf{B}=(0, B, 0))$, is inhomogeneous in the $z$-direction. A Gaussian profile of the field is assumed

$$
B(z)=B_{0} \exp \left[-\frac{\left(z-z_{0}\right)^{2}}{2 \sigma_{B}^{2}}\right],
$$

where $B_{0}$ is the maximum value at $z=z_{0}$ and $\sigma_{B}$ scales the field variation. The driver which maintains the plasma of the discharge is shaped by a power input localized in the region of the weak magnetic field before the filter. A super-Gaussian profile of the power input variation in the $z$-direction is assumed

$$
P_{w}(z)=P_{W 0} \exp \left[-\frac{1}{2}\left(\frac{z}{\sigma_{P}}\right)^{2 m}\right],
$$

with a maximum value $P_{W 0}$ at $z=0$ and $\sigma_{P}$ scaling its changes. With the Maxwellian distribution function used for calculating the rate coefficients of the different processes in the plasma, a rf-discharge is assumed as a driver.

Since the transport processes in the plasma are in all, three, directions (along the $x$-, $y$ - and $z$-axes), a $3 \mathrm{D}$ model - as it is developed here - is that providing a complete description of the magnetic filter 
operation. However, this study also stresses that the 2D model describing the changes - due to the magnetic field - of the plasma parameters in the (x-z)-plane is good enough. (As figure 1(a) shows, the (x$z$ )-plane is perpendicular to the magnetic field and it is located in the middle between the filter magnets.) Moreover, the 1D model which describes the changes of the plasma parameters along the length $z$ of the discharge vessel, perpendicularly to the magnetic field, appears very useful for giving the indications about the main trends in the operation of the filter.
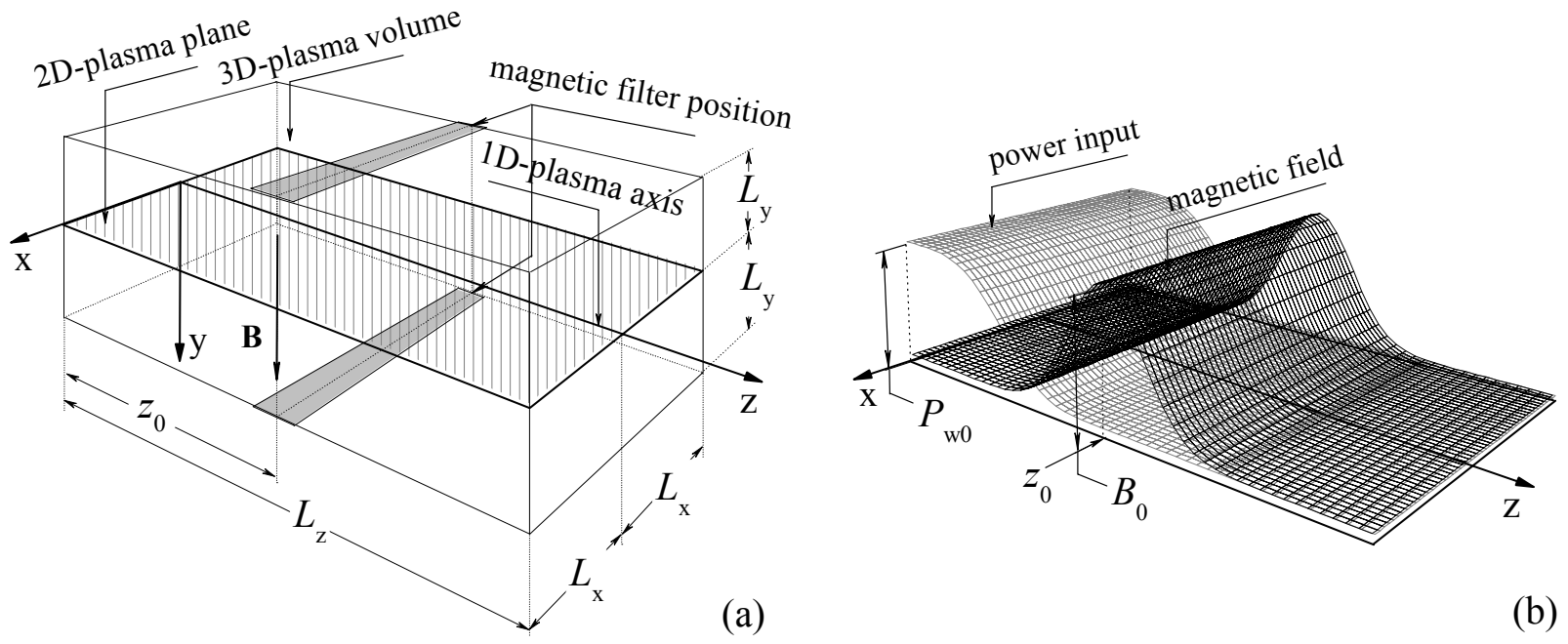

Figure 1. Configuration of the plasma volume in (a) and an illustration of the $z$-variations of the magnetic field and of the power input in (b).

\subsection{Charged particle description}

The fluid-plasma model of the operation of the filter in low-pressure gas discharges plasmas, presented here, is based on the continuity equations of the charged particles

their momentum equations

$$
\operatorname{div}\left(n_{\alpha} \mathbf{v}_{\alpha}\right)=\frac{\delta n_{\alpha}}{\delta t}
$$

$$
Z_{\alpha} e n_{\alpha}\left(\mathbf{E}+\mathbf{v}_{\alpha} \times \mathbf{B}\right)-\operatorname{grad}\left(n_{\alpha} T_{\alpha}\right)-\left(\sum_{j} \mu_{\alpha j} v_{\alpha j}\right) n_{\alpha} \mathbf{v}_{\alpha}=0
$$

the electron energy balance

$$
\operatorname{div} \mathbf{Q}_{e}=P_{w}-P_{\text {coll }}
$$

and the Poisson equation

$$
\Delta \phi=\frac{e}{\varepsilon_{0}}\left(n_{e}-\sum_{l=1}^{3} n_{i_{l}}\right)
$$

In (3), (4) and (6), $\alpha$ refers to the different types of charged particles (electrons $\alpha=e$ and ions $\alpha=i_{l}$ ) and $Z_{\alpha}$ denotes the sign of their charges, $n_{\alpha}, \mathbf{v}_{\alpha}$ and $T_{\alpha}$ are, respectively, their densities, directed velocities and temperatures (in energy units), $\mathbf{E}$ is the dc electric field formed - due to the charged particle fluxes to the discharge walls - in the low-pressure discharges and $\phi$ is its potential $(\mathbf{E}=-\operatorname{grad} \phi), \quad v_{\alpha j}$ are the elastic collision frequencies of the charged particles $(\alpha)$ with neutrals $(j)$, $\mu_{\alpha j}=m_{\alpha} m_{j} /\left(m_{\alpha}+m_{j}\right)$ are the reduced masses at elastic collisions, $m_{\alpha}$ and $m_{j}$ are the masses of the charged and neutral species, $\varepsilon_{0}$ is the vacuum permittivity and $\left(\delta n_{\alpha} / \delta t\right)$ describes the production and losses of charged particles by collisions in the plasma volume. Presence of different types of positive ions 
$\left(i_{l}\right)$ and neutrals $(j)$ is assumed having in mind the hydrogen discharges where $\mathrm{H}^{+}, \mathrm{H}_{2}^{+}$and $\mathrm{H}_{3}^{+}$are the positive ions and hydrogen atoms $\mathrm{H}$ and molecules $\mathrm{H}_{2}$ are the neutral species. In (5),

$$
\mathbf{Q}_{e}=\mathbf{q}_{e}+\frac{5}{2} n_{e} T_{e} \mathbf{v}_{e}
$$

is the electron energy flux, including the thermal flux $\mathbf{q}_{e}$ (the conductive flux) and the flux of thermal energy and pressure force work carried by the directed velocity (i.e., the convective flux as given by the second term in (7)), and $P_{W}$ and $P_{\text {coll }}$ are, respectively, the power input (2) applied for the discharge maintenance and the electron energy losses in collisions. The equation determining the thermal flux $\mathbf{q}_{e}$ of the electrons is [28]:

$$
\frac{5}{2} \frac{n_{e} T_{e}}{m_{e}} \operatorname{grad} T_{e}+\frac{e}{m_{e}}\left(\mathbf{q}_{e} \times \mathbf{B}\right)=-v_{e} \mathbf{q}_{e}
$$

where $v_{e}$ is the total elastic electron-neutral collision frequency (in the case of hydrogen discharges, electron collisions with hydrogen atoms and molecules). The electron energy losses at the wall are taken into account in the boundary conditions given at the end of this subsection.

The velocities of the charged particles along $\left(\mathbf{v}_{\| \alpha}\right)$ and across $\left(\mathbf{v}_{\perp \alpha}\right)$ the magnetic field obtained from (4) are, respectively:

$$
\begin{gathered}
\mathbf{v}_{\| \alpha}=Z_{\alpha} b_{\| \alpha} \mathbf{E}_{\|}-D_{\| \alpha} \frac{1}{n_{\alpha}} \operatorname{grad}_{\|} n_{\alpha}-D_{\| \alpha}^{T} \frac{1}{T_{\alpha}} \operatorname{grad}_{\|} T_{\alpha} \\
\mathbf{v}_{\perp \alpha}=Z_{\alpha} b_{\perp \alpha} \mathbf{E}_{\perp}-D_{\perp \alpha} \frac{1}{n_{\alpha}} \operatorname{grad}_{\perp} n_{\alpha}-D_{\perp \alpha}^{T} \frac{1}{T_{\alpha}} \operatorname{grad}_{\perp} T_{\alpha} \\
+b_{d \alpha}\left(\mathbf{E}_{\perp} \times \mathbf{h}\right)+Z_{\alpha} D_{d \alpha} \frac{1}{n_{\alpha}}\left(\mathbf{h} \times \operatorname{grad}_{\perp} n_{\alpha}\right)+Z_{\alpha} D_{d \alpha}^{T} \frac{1}{T_{\alpha}}\left(\mathbf{h} \times \operatorname{grad}_{\perp} T_{\alpha}\right)
\end{gathered}
$$

where $\mathbf{h}$ is the unit vector along the magnetic field $(\mathbf{h}=\mathbf{B} / B)$,

$$
\begin{gathered}
b_{\| \alpha}=\frac{e}{\mu_{\alpha} v_{\alpha}}, \quad b_{\perp \alpha}=\frac{b_{\| \alpha}}{1+\left(\frac{m_{\alpha} \Omega_{\alpha}}{\mu_{\alpha} v_{\alpha}}\right)^{2}} \\
D_{\| \alpha}=\frac{T_{\alpha}}{\mu_{\alpha} v_{\alpha}}, \quad D_{\perp \alpha}=\frac{D_{\| \alpha}}{1+\left(\frac{m_{\alpha} \Omega_{\alpha}}{\mu_{\alpha} v_{\alpha}}\right)^{2}}
\end{gathered}
$$

are, respectively, the mobilities and the diffusion coefficients along the magnetic field and in the transverse direction and

$$
b_{d \alpha}=\frac{m_{\alpha} \Omega_{\alpha}}{\mu_{\alpha} v_{\alpha}} b_{\perp \alpha}, \quad D_{d \alpha}=\frac{m_{\alpha} \Omega_{\alpha}}{\mu_{\alpha} v_{\alpha}} D_{\perp \alpha}
$$

are the mobility and the diffusion coefficient related, respectively, to the $(\mathbf{E} \times \mathbf{B})$-drift and the diamagnetic drift; $\Omega_{\alpha}=e B / m_{\alpha}$ is the gyro-frequency, and the notation $\mu_{\alpha} v_{\alpha}=\sum_{j} \mu_{\alpha j} v_{\alpha j}$ has been used. For the thermal diffusion coefficients, one has $D_{\| \alpha}^{T}=D_{\| \alpha}, D_{\perp \alpha}^{T}=D_{\perp \alpha}$ and $D_{d \alpha}^{T}=D_{d \alpha}$.

With components of charged particle fluxes given by

$$
\begin{aligned}
\Gamma_{x \alpha}= & -Z_{\alpha} n_{\alpha} b_{\perp \alpha} \partial_{x} \phi-D_{\perp \alpha} \partial_{x} n_{\alpha}-D_{\perp \alpha}^{T} \frac{n_{\alpha}}{T_{\alpha}} \partial_{x} T_{\alpha} \\
& +n_{\alpha} b_{d \alpha} \partial_{z} \phi+Z_{\alpha} D_{d \alpha} \partial_{z} n_{\alpha}+Z_{\alpha} \frac{n_{\alpha}}{T_{\alpha}} D_{d \alpha}^{T} \partial_{z} T_{\alpha}
\end{aligned}
$$




$$
\begin{gathered}
\Gamma_{y \alpha}=-Z_{\alpha} n_{\alpha} b_{\| \alpha} \partial_{y} \phi-D_{\| \alpha} \partial_{y} n_{\alpha}-\frac{n_{\alpha}}{T_{\alpha}} D_{\| \alpha}^{T} \partial_{y} T_{\alpha} \\
\Gamma_{z \alpha}=-Z_{\alpha} n_{\alpha} b_{\perp \alpha} \partial_{z} \phi-D_{\perp \alpha} \partial_{z} n_{\alpha}-\frac{n_{\alpha}}{T_{\alpha}} D_{\perp \alpha}^{T} \partial_{z} T_{\alpha} \\
-n_{\alpha} b_{d \alpha} \partial_{x} \phi-Z_{\alpha} D_{d \alpha} \partial_{x} n_{\alpha}-Z_{\alpha} \frac{n_{\alpha}}{T_{\alpha}} D_{d \alpha}^{T} \partial_{x} T_{\alpha},
\end{gathered}
$$

the continuity equations take the compact form

$$
\operatorname{div} \boldsymbol{\Gamma}_{\alpha}=\frac{\delta n_{\alpha}}{\delta t}
$$

where

$$
\boldsymbol{\Gamma}_{\alpha}=-Z_{\alpha} n_{\alpha} \hat{\mathbf{b}}_{\alpha} \cdot \operatorname{grad} \phi-\hat{\mathbf{D}}_{\alpha} \cdot \operatorname{grad} n_{\alpha}-\frac{n_{\alpha}}{T_{\alpha}} \hat{\mathbf{D}}_{\alpha}^{T} \cdot \operatorname{grad} T_{\alpha}
$$

with

and

$$
\begin{aligned}
\hat{\mathbf{b}}_{\alpha} & =\left[\begin{array}{ccc}
b_{\perp \alpha} & 0 & -Z_{\alpha} b_{d \alpha} \\
0 & b_{\| \alpha} & 0 \\
Z_{\alpha} b_{d \alpha} & 0 & b_{\perp \alpha}
\end{array}\right], \\
\hat{\mathbf{D}}_{\alpha} & =\left[\begin{array}{ccc}
D_{\perp \alpha} & 0 & -Z_{\alpha} D_{d \alpha} \\
0 & D_{\| \alpha} & 0 \\
Z_{\alpha} D_{d \alpha} & 0 & D_{\perp \alpha}
\end{array}\right]
\end{aligned}
$$

$$
\hat{\mathbf{D}}_{\alpha}^{T}=\hat{\mathbf{D}}_{\alpha}
$$

being the mobility-, diffusion- and thermal diffusion- tensors.

The components of the thermal flux of the electrons along and across the magnetic field obtained from (8) are, respectively:

where

$$
\begin{gathered}
\mathbf{q}_{\| e}=-\chi_{\| e} \operatorname{grad}_{\|} T_{e} \\
\mathbf{q}_{\perp e}=-\chi_{\perp e} \operatorname{grad}_{\perp} T_{e}-\chi_{d e}\left(\mathbf{h} \times \operatorname{grad}_{\perp} T_{e}\right)
\end{gathered}
$$

$$
\chi_{\| e}=\frac{5}{2} n_{e} D_{\| e}, \quad \chi_{\perp e}=\frac{\chi_{\| e}}{1+\left(\frac{\Omega_{e}}{v_{e}}\right)^{2}}
$$

are the thermal conductivity coefficients in the directions parallel and perpendicular to the magnetic field and

$$
\chi_{d e}=\frac{\Omega_{e}}{v_{e}} \chi_{\perp e}
$$

is the thermal conductivity coefficient related to the diamagnetic drift.

Thus, the components - along the three axes $(x, y$ and $z)$ - of the total flux of the electron energy present in (5) are:

$$
\begin{gathered}
Q_{x e}=-\chi_{\perp e} \partial_{x} T_{e}-\chi_{d e} \partial_{z} T_{e}+\frac{5}{2} n_{e} T_{e} v_{x e} \\
Q_{y e}=-\chi_{\| e} \partial_{y} T_{e}+\frac{5}{2} n_{e} T_{e} v_{y e} \\
Q_{z e}=-\chi_{\perp e} \partial_{z} T_{e}+\chi_{d e} \partial_{x} T_{e}+\frac{5}{2} n_{e} T_{e} v_{z e} .
\end{gathered}
$$

Written in a vector form, the electron energy flux is: 


$$
\mathbf{Q}_{e}=-\hat{\chi}_{e} \cdot \operatorname{grad} T_{e}+\frac{5}{2} T_{e} \boldsymbol{\Gamma}_{e}
$$

where

$$
\hat{\chi}_{e}=\left[\begin{array}{ccc}
\chi_{\perp e} & 0 & \chi_{d e} \\
0 & \chi_{\| e} & 0 \\
-\chi_{d e} & 0 & \chi_{\perp e}
\end{array}\right]
$$

is the thermal conductivity tensor and $\boldsymbol{\Gamma}_{e}$ is the flux (12b) of the electrons.

Now the boundary conditions should be specified. The charged particle fluxes parallel to the magnetic field, i.e. the fluxes along the $y$-direction $(\mathbf{B} \| \mathbf{y})$ are anyhow not influenced by the magnetic field and, thus, the boundary conditions at $y= \pm L_{y}$ are those, well-known from the literature [e.g., 29-32]:

$$
\begin{gathered}
\Gamma_{e y}=\frac{1}{4} v_{t h, e} n_{e} \\
\Gamma_{i_{l} y}=n_{i_{l}} b_{\| i_{l}} \frac{\partial \phi}{\partial y}+\frac{1}{2} v_{t h, i_{l}} n_{i_{l}},
\end{gathered}
$$

respectively, for electrons and ions. In (18), $v_{t h, \alpha}=\sqrt{8 T_{\alpha} / \pi m}$ is the thermal velocity of the corresponding types of particles (electrons and ions) and " $l$ " marks the different types of ions. The boundary condition (18a) for the electron flux corresponding to Boltzman distribution of the electron density and a Maxwellian electron energy distribution is for a collisionless sheath $[29,30]$. The electric field is present in the boundary condition for the flux of the ions since their motion is in an accelerating field [30,31].

The magnetic field, influencing the charged particle fluxes perpendicularly to B ((11a) and (11c)), modifies also the boundary conditions at the wall surfaces parallel to the field. Following the procedure described in Ref. [33], the boundary conditions at $x= \pm L_{x}$ and $z=L_{z}$, i.e. the fluxes perpendicular to the magnetic field, appear in the form:

$$
\begin{gathered}
\Gamma_{e x, z}=\frac{1}{1+\left(\frac{m_{e} \Omega_{e}}{\mu_{e} v_{e}}\right)^{2}}\left(\frac{1}{4} v_{t h, e} n_{e}\right) \\
\Gamma_{i_{l} x, z}=\frac{1}{1+\left(\frac{m_{i_{l}} \Omega_{i_{l}}}{\mu_{i_{l}} v_{i_{l}}}\right)^{2}}\left(n_{i_{l}} b_{\| i_{l}} \nabla_{x, z} \phi+\frac{1}{2} v_{t h, i_{l}} n_{i_{l}}\right) .
\end{gathered}
$$

Expressions (19a) and (19b) clearly show - by the first multipliers therein - the reduction by the magnetic field of the fluxes without an external magnetic field given by the second multipliers in (19a) and (19b).

With the zero thermal flux at the discharge walls usually considered [28,32,34], the boundary condition for the electron energy flux at the walls $\left(x= \pm L_{x}, y= \pm L_{y}\right.$ and $\left.z=L_{z}\right)$ is

$$
\begin{gathered}
Q_{e y}=\frac{5}{2} T_{e}\left(\frac{1}{4} v_{t h, e} n_{e}\right) \\
Q_{e x, z}=\left(\frac{5}{2} T_{e}\right) \frac{1}{1+\left(\frac{m_{e} \Omega_{e}}{\mu_{e} v_{e}}\right)^{2}}\left(\frac{1}{4} v_{t h, e} n_{e}\right) .
\end{gathered}
$$

The second multiplier in (20b) shows the reduction of the fluxes due to the magnetic field.

The value of the potential $\phi$ of the dc electric field at $x= \pm L_{x}, y= \pm L_{y}$ and $z=L_{z}$ (metal walls) is fixed to zero:

$$
\left.\phi\right|_{\text {walls }}=0
$$


The boundary conditions at $z=0$, where the power input is located, are in an accordance with the symmetry assumed here: $\left.(\partial \phi / \partial z)\right|_{z=0}=0$ and zero charged-particle fluxes.

\subsection{Collisional processes specifying hydrogen discharges}

The equations in Subsection 2.1 are common notwithstanding the type of the gas in the discharge. The latter is specified by the processes of production and destruction of charged particles (i.e., by the $\left(\delta n_{\alpha} / \delta t\right)$-term in (3)), the processes of electron energy losses in collisions (the term $P_{\text {coll }}$ in (5)) as well as by the description of the neutral gas (or its components). Regarding the use of the magnetic filter for electron cooling in the negative ion sources developed for fusion-plasma heating, discharge maintenance in a hydrogen gas is specified. Electrons and the three types of positive ions $\left(\mathrm{H}^{+}, \mathrm{H}_{2}^{+}\right.$and $\left.\mathrm{H}_{3}^{+}\right)$are the charged particles involved in the model. Looking for the general trends of the changes of the spatial distribution of the main plasma parameters and the mechanisms of the magnetic filter operation, negative hydrogen ions are not considered. However, due to the high efficiency of the dissociation in hydrogen discharges, both hydrogen atoms $\mathrm{H}$ and molecules $\mathrm{H}_{2}$ are taken into account as completing the neutral gas in the discharge. For determining the concentrations $N_{a}$ of the atomic hydrogen component, its balance equation

$$
\operatorname{div}\left(-D_{a} \operatorname{grad} N_{a}\right)=\frac{\delta N_{a}}{\delta t}
$$

is added to the balance equations (3)-(5) of the charged particles. In (22), $D_{a}$ is the corresponding diffusion coefficient and $\left(\delta N_{a} / \delta t\right)$ describes the production and losses of hydrogen atoms by collisions in the discharge volume. The concentration $N_{m}$ of the molecular hydrogen is obtained from the equation of state

$$
p=T_{g}\left(N_{a}+N_{m}\right)
$$

where $p$ is the gas pressure and $T_{g}$ is the gas temperature, in energy units.

The following processes in the plasma volume, usually considered [35-38] as being the main contributors to the production and destruction of charged and neutral-gas species, are taken into account in the particle balance equations (3) and (22): (i) ionization of molecules $\left(e+\mathrm{H}_{2}\left(\mathrm{X}^{1} \Sigma_{g}^{+}\right) \rightarrow e+\mathrm{H}_{2}^{+}(v)+e\right)$, (ii) dissociation of molecules $\left(e+\mathrm{H}_{2}\left(\mathrm{X}^{1} \Sigma_{g}^{+}\right) \rightarrow e+\mathrm{H}(1 s)+\mathrm{H}(1 s)\right)$, (iii) dissociation of $\mathrm{H}_{2}^{+}$-ions $\left(e+\mathrm{H}_{2}^{+}(v) \rightarrow e+\mathrm{H}^{+}+\mathrm{H}(1 s)\right)$, (iv) heavy particle collisions $\left(\mathrm{H}_{2}^{+}+\mathrm{H}_{2} \rightarrow \mathrm{H}_{3}^{+}+\mathrm{H}\right)$, (v) atom ionization $\left(e+\mathrm{H}(1 s) \rightarrow e+\mathrm{H}^{+}+\mathrm{e}\right)$, (vi) dissociative recombination of $\mathrm{H}_{3}^{+}$-ions $\left(e+\mathrm{H}_{3}^{+} \rightarrow \mathrm{H}+\mathrm{H}+\mathrm{H}\right)$ and (vii) dissociation of $\mathrm{H}_{3}^{+}$-ions $\left(e+\mathrm{H}_{3}^{+} \rightarrow \mathrm{e}+\mathrm{H}^{+}+2 \mathrm{H}\right)$. The rate coefficients of these reactions are $k_{j}$ with $j=1-7$, according to their numbering in the above list, and " $v$ " denotes vibrationally excited species. Thus, the production and losses in the plasma volume of electrons, positive ions (with concentration $n_{i_{1}}, n_{i_{2}}$ and $n_{i_{3}}$ ) and hydrogen atoms are:

$$
\begin{gathered}
\frac{\delta n_{e}}{\delta t}=k_{1} N_{m} n_{e}+k_{5} N_{a} n_{e}-k_{6} n_{i_{3}} n_{e} \\
\frac{\delta n_{i_{1}}}{\delta t}=k_{3} n_{i_{2}} n_{e}+k_{5} N_{a} n_{e}+k_{7} n_{i_{3}} n_{e} \\
\frac{\delta n_{i_{2}}}{\delta t}=k_{1} N_{m} n_{e}-k_{3} n_{i_{2}} n_{e}-k_{4} n_{i_{2}} N_{m} \\
\frac{\delta n_{i_{3}}}{\delta t}=k_{4} n_{i_{2}} N_{m}-k_{6} n_{i_{3}} n_{e}-k_{7} n_{i_{3}} n_{e} \\
\frac{\delta N_{a}}{\delta t}=2 k_{2} N_{m} n_{e}+k_{3} n_{i_{2}} n_{e}+k_{4} n_{i_{2}} N_{m}-k_{5} N_{a} n_{e}+3 k_{6} n_{i_{3}} n_{e}+2 k_{7} n_{i_{3}} n_{e} .
\end{gathered}
$$

The boundary condition for the hydrogen atoms at $z=L_{z}, x= \pm L_{x}$ and $y= \pm L_{y}$ 


$$
\left.\Gamma_{a}\right|_{\text {walls }}=\frac{1}{2} \gamma v_{t h, a} N_{a}
$$

accounts for their recombination at the walls; $\gamma$ is the wall recombination efficiency, $\boldsymbol{\Gamma}_{a}=-D_{a} \operatorname{grad} N_{a}$ is the flux of the hydrogen atoms and $v_{t h, a}=\sqrt{8 T_{g} / \pi m_{a}}$ is their thermal velocity; a zero flux is the boundary condition at $z=0$.

The electron energy losses $P_{\text {coll }}$ in collisions taken into account are the same as in the model in Ref. [38], including: (i) losses for atom excitation and ionization, (ii) losses for excitation of vibrational and singlet states of the molecules as well as for their dissociation and ionization, and (iii) losses in elastic collisions with atoms and molecules.

The data for the coefficients of the processes included in the particle- and the electron-energy- balance as well as the frequencies of elastic collisions are taken as described in Ref. [38]. The rate coefficients of all the electron impact processes both in the particle- and electron-energy- balance are taken from Janev et al [39], calculated there for a Maxwellian electron energy distribution. The rate coefficient of reaction (iv) is the same as in $[35,37,40]$. The frequencies of elastic collisions of ions with neutrals are calculated by using data from [28,41-43]. For obtaining the elastic electron-neutral collision frequency, and its temperature dependence, the data for the cross sections from Ref. [44] have been used. The diffusion coefficient of the hydrogen atoms is according to Ref. [45].

\section{Results and discussions}

Magnetic filter operation in hydrogen discharges is considered. The results from the model describe the influence of the magnetic field on the spatial distribution of the plasma parameters: electron temperature $T_{e}$ and concentration $n_{e}$, concentrations $n_{i_{1}}, n_{i_{2}}$ and $n_{i_{3}}$ of the three types of positive ions (respectively, $\mathrm{H}^{+}, \mathrm{H}_{2}^{+}$and $\mathrm{H}_{3}^{+}$), concentrations $N_{a}$ and $N_{m}$ of the neutral species (respectively, atoms and molecules) and the potential $\phi$ of the dc electric field. They are obtained as numerical solutions of the set of equations completed by (3), written for the four types of charged particles with the corresponding charged particle fluxes (11) replaced therein, (5), (6) and (22); the boundary conditions are as given in Subsection 2.1. Results obtained within 1D-, 2D - and 3D-models are presented. The 1D-model shows the variations of the plasma parameters in the $z$-direction (figure 1) which is along the discharge vessel, perpendicularly to the external magnetic field. The 2D-model describes variations of the plasma parameters also in the $x$-direction, added to the $z$-one. Thus, ignoring fluxes and changes of the plasma parameters along the magnetic field (the $y$-direction), the 2D-model presents the structure of the discharge in the $(x-z)$-plane located in the middle of the discharge vessel (figure 1), perpendicularly to the magnetic field. The 3D-model describes the space distribution of the plasma parameters both along and across the magnetic field.

The size of the discharge chamber and, respectively, of the domain setting for solving numerically the set of equations is: a length of $L_{z}=20 \mathrm{~cm}$ and a width and a height, respectively, of $2 L_{x}=20 \mathrm{~cm}$ and $2 L_{y}=20 \mathrm{~cm}$. The position of the magnetic filter is the same, in the three models, with a maximum value of the magnetic field located at $z=10 \mathrm{~cm}$. The values of $\sigma_{P}=4.729 \mathrm{~cm}$ and $\sigma_{B}=1.581 \mathrm{~cm}$ characterizing, respectively, the widths of the variation of the power input (with a maximum located at $z=0 \mathrm{~cm})$ and of the magnetic field are also kept the same. The values of the other external parameters are, as follows: $p=5$ mTorr of the gas pressure, $P_{W 0}=10^{4} \mathrm{~W} / \mathrm{m}^{3}$ of the maximum value of the power (2) applied for the discharge maintenance with $m=2$ as a parameter of its super-Gaussian shape and $T_{g}=2.58 \times 10^{-2} \mathrm{eV}$ (i.e. $300 \mathrm{~K}$ ) of the gas temperature; the temperature of the ions is also constant, the same as of the neutrals $\left(T_{i} \equiv T_{g}\right)$. In the $2 \mathrm{D}$-model, the value of the magnetic field is varied up to $B_{0}=100 \mathrm{G}$. Results for a zero magnetic field $\left(B_{0}=0 \mathrm{G}\right)$ are also shown, regarding the discussions on the filter operation. For the discharge conditions considered, magnetic field values even below $B_{0}=10 \mathrm{G}$ ensure strong magnetization of the electrons $\left(v_{e} / \Omega_{e}<1\right)$. In opposite, even for the highest value 
$B_{0}=100 \mathrm{G}$ of the magnetic field taken here, the magnetic field does not affect the ion dynamics $\left(v_{i} / \Omega_{i}>1\right)$.

The obtained results show that elastic electron-neutral collisions govern the transport coefficients of the electrons, as it has been supposed by taking the momentum equations of the charged particles in the form of equation (4). In fact, under the conditions of the 2D- and 3D- cases, the electron-electron collision frequency $v_{e-e}$ is at least two orders of magnitude lower than the electron-neutral collision frequency. In the case of the 1D-model considered, Coulomb collisions could start playing a role in the region of the magnetic filter for magnetic field values above $50 \mathrm{G}$. By that reason, in this case $B_{0}$ is varied up to that value $\left(B_{0} \leq 50 \mathrm{G}\right)$. Comparison of the values of the elastic electron-atom and electronmolecule collisions shows that electron elastic collisions with molecule predominate over collisions with atoms. The latter is in agreement with the results in [46] for a low degree of dissociation when the electron density is comparatively low like here.

Since the power input is localized, strong effects of nonlocality in the discharge maintenance should be expected. Plasma existence in the discharge vessel outside the region of the power deposition should result from nonlocal electron heating and electron energy fluxes, i.e. from thermal conductivity effects. Moreover, since the magnetic field is outside the region of the power input this flux should be strongly influenced - in fact, suppressed - by the magnetic field. Due to the suppressed thermal conductivity, strong modification of the whole structure of the discharge could be expected.

\subsection{D-model description}

The 1D-model considers the modifications - due to the magnetic filter - of the plasma parameters in the $z$-direction (figure 1), ignoring the changes in the $x$ - and $y$-directions. Thus, the charged-particle and electron-energy fluxes are only along the $z$-axis. The last three terms in (11c) and the second term in (16c) drop out and, thus, there is no effects of $(\mathbf{E} \times \mathbf{B})$ - and diamagnetic-drifts. Therefore, mobility in the dc field, diffusion and thermal diffusion, all of them across the magnetic field, as described by the first three terms in (11c), determine the charged particle fluxes. The electron energy flux is due to the thermal conductivity across the magnetic field (the first term in (16c)) and thermal energy and pressure force work carried by the directed velocity along the $z$-axis (given by the last term in (16c)).

The results from the 1D-model shown in figure 2 are for different values of the magnetic field in the center of the filter: values up to $B_{0}=50 \mathrm{G}$, starting from the case without magnetic field.
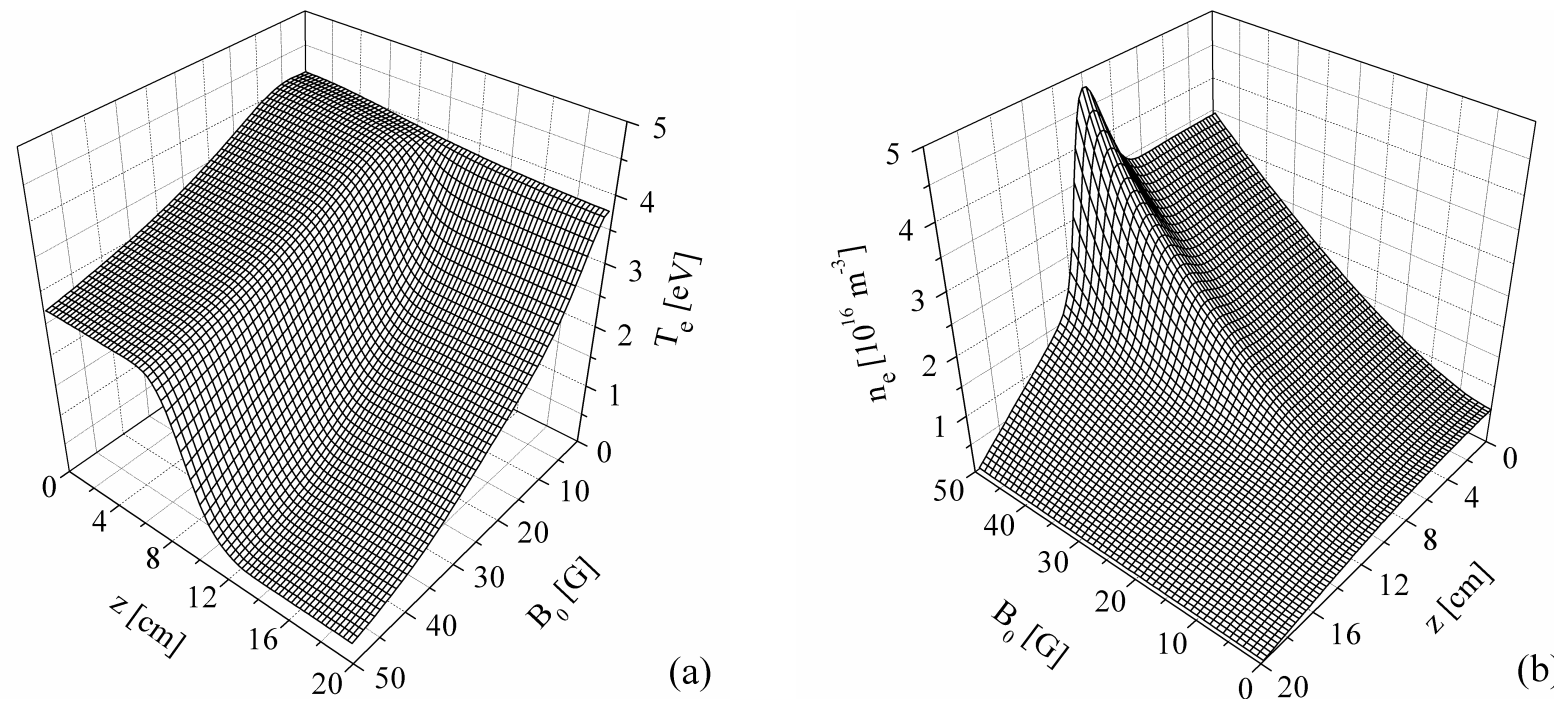

Figure 2. Results from the 1D-model: Axial distribution of the electron temperature (a) and density (b) for varying values of the magnetic field $B_{0}$ at the center of the filter.

The obtained results show that not only collisions but also the convective flux (the second term in equation (7)) appears as losses in the electron energy balance (7). This hold both without and with 
magnetic field applied and over the total length of the discharge chamber. In fact, the plasma production outside the region of the power input results from energy transfer through thermal conductivity. The large value of the coefficient of thermal conductivity when the magnetic field is zero $\left(\chi_{\| e}\right.$ in (15a)) ensures a high thermal flux $\left(\left.q_{z e}\right|_{B=0}=-\chi_{\| e}\left(\mathrm{~d} T_{e} / \mathrm{d} z\right)\right)$ and the axial decrease of the electron temperature (figure 2(a)) is very slight. The reduction of the thermal conductivity coefficient ( $\chi_{\perp e}$ in (15a)) with the increase of the magnetic field is responsible for a reduction of the thermal flux (the first term in (16c)). Thus, imposing the magnetic filter leads to suppressed nonlocality of the electron heating. The result is an electron cooling showed by the sharp axial drop of the electron temperature. Therefore, the 1D model outlines the suppression of the thermal conductivity by the magnetic field as a mechanism of the operation of the filter. The total decrease of $T_{e}$ with increasing $B_{0}$ is due to reduced diffusion losses to the walls (due to the decrease of $D_{\perp e}$ in (10b)) which leads to the increase of the plasma density in the filter region shown in figure 2(b).

With the almost constant $T_{e}$ for $B_{0}=0$, diffusion losses with coefficient $D_{\| e}(10 \mathrm{~b})$ determine the usual smooth profile of the electron density over the total length of the discharge. In fact, the density distribution is close to a cosine-type of profile, as it should be expected [28,29]. With increasing $B_{0}$, the electron density increases due to the reduction of the diffusion losses (according to $D_{\perp \alpha}$ in (10b)). For $B_{0}$ large enough, the axial profile of the electron density becomes nonmonotonic with a formation of a maximum of $n_{e}$ in the region of the filter. According to (11c) written in the 1D model, this is due to the axial drop of $T_{e}$ bringing into play thermal diffusion (the third term in (11c)) acting together with the diffusion. The $T_{e}(z)$-decrease leads to formation of a forward thermal-diffusion flux $\left.\Gamma_{z e}\right|_{T D}=-D_{\perp e}\left(n_{e} / T_{e}\right)\left(\mathrm{d} T_{e} / \mathrm{d} z\right)$. On the other hand, the diffusion flux is lowered, due to the magnetic field $\left(\left.\Gamma_{z e}\right|_{D}=-D_{\perp e}\left(\mathrm{~d} n_{e} / \mathrm{d} z\right)\right)$. This leads to an accumulation of electrons in the region of the filter and to the formation of the maximum of $n_{e}$ shown in figure 2(b).

With the values of $n_{e}$ and $T_{e}$ obtained within the 1D-model, the density $n_{i_{1}}$ of the $\mathrm{H}^{+}$-ions is the highest one, among the ion species in the discharge. Its axial variation follows the axial variation (figure 2(b)) of $n_{e}$. The axial variations of the concentrations of $\mathrm{H}_{2}^{+}$- and $\mathrm{H}_{3}^{+}$-ions are also in the same trends. The accumulation of positive ions in the filter region could be associated with the condition of quasineutrality in the plasma volume. The latter is in accordance with the obtained axial variation of the potential of the dc field: slight - except for very small $B_{0}$-values - axial decrease of $\phi$ in the plasma volume, with a strong drop at the discharge walls, as it is expected. The axial changes of $\phi$ for low $B_{0}$ are comparatively strong, due to the low values of the plasma density (figure $2(a)$ ). With increasing $B_{0}$, $\phi$ decreases in a correlation with the corresponding decrease of $T_{e}$ (figure 2(a)).

The drop of the electron temperature and the increase of the plasma density in the region of the filter are the main results obtained within the $1 \mathrm{D}$ model. The model also shows that reduction of the thermal flux of the electrons, leading to suppression of the nonlocal heating, as well as an importance of the thermal diffusion flux, acting together with the diffusion flux, are the mechanisms determining the redistribution of the plasma parameters caused by the magnetic field.

\section{2. $2 D$-model description}

The results from the 2D-model presented here describe the spatial distribution of the plasma parameters in the $(x-z)$-plane (figure 1), i.e. perpendicularly to the magnetic field, ignoring the fluxes along the magnetic field. With the second $(x-)$ direction taken into account, effects related to the $(\mathbf{E} \times \mathbf{B})$-drift and to the diamagnetic drift (as described by the three last terms in (11a) and (11c)) are expected to show evidence, in addition to the effects described within the 1D model. Respectively, the thermal fluxes related to the diamagnetic drift (the second terms in (16a) and (16c)) are also sources of additional modification of the plasma parameters. 
Results comparing the spatial distribution of the plasma parameters obtained for $B_{0}=0 \mathrm{G}$ and $B_{0}=50 \mathrm{G}$ are discussed. The spatial variations shown here (figures 3-6) are of the electron temperature $T_{e}$ and density $n_{e}$, of the of the potential $\phi$ of the dc electric field and of the concentration of the hydrogen atoms. The spatial variations of the concentrations of the ions and of the molecules, also resulting from the model, are briefly discussed.

Figure 3 presents the spatial redistribution of $T_{e}$ caused by the magnetic filter. The slow axial variation of $T_{e}$ for absent magnetic field (figure 3(a)) is slightly bigger - compared to the 1D model due to the additional losses in the $x$-direction taken into account now. The $x$-variation of $T_{e}$ in the vicinity of $z=0$ is related to the manner of the power input deposition. Since the electron density decreases (figure (4a)) towards the walls and the power input (2) is homogeneous in the $x$-direction, $T_{e}$ should increase towards the walls $x= \pm L_{x}$, as figure 3(a) shows. The strong effect of the filter could be seen in figure 3(b). The electron temperature drops from $7.5 \mathrm{eV}$ to $3 \mathrm{eV}$ forming a groove where the value of the temperature is $2.5 \mathrm{eV}$. Although the drop of $T_{e}$ is the effect predicted also by the 1D model (figure 2(a)) here, in the 2D model, the picture is quite more complicated (figure 3(b)). Effects due to the thermal fluxes related to the diamagnetic drift and their combined action with thermal fluxes in a transverse magnetic field are responsible for this. Reduction of the thermal conductivity coefficient $\chi_{\perp e}$ (15a) and suppressed nonlocality of the electron heating due to the magnetic field cause the axial decrease of $T_{e}$, like in the 1D model. However, the 2D-model gives the after-effects of this on the distribution in the $x$ direction: A thermal flux related to the diamagnetic drift appears in the $x$-direction (as described by the second term in (16a)) due to the $z$-decrease of $T_{e}$. This causes the increase of $T_{e}$ in the $x$-direction (figure 3(b)). The latter leads to a thermal flux in the $z$-direction (described by the second term in (16c)) which is again related to a diamagnetic drift. This flux causes electron heating behind the filter and the formation of a minimum (or of the groove) in the region of the filter. The formation of the groove leads itself to an inverse thermal flux in the $z$-direction, from the region behind the filter to the filter region.
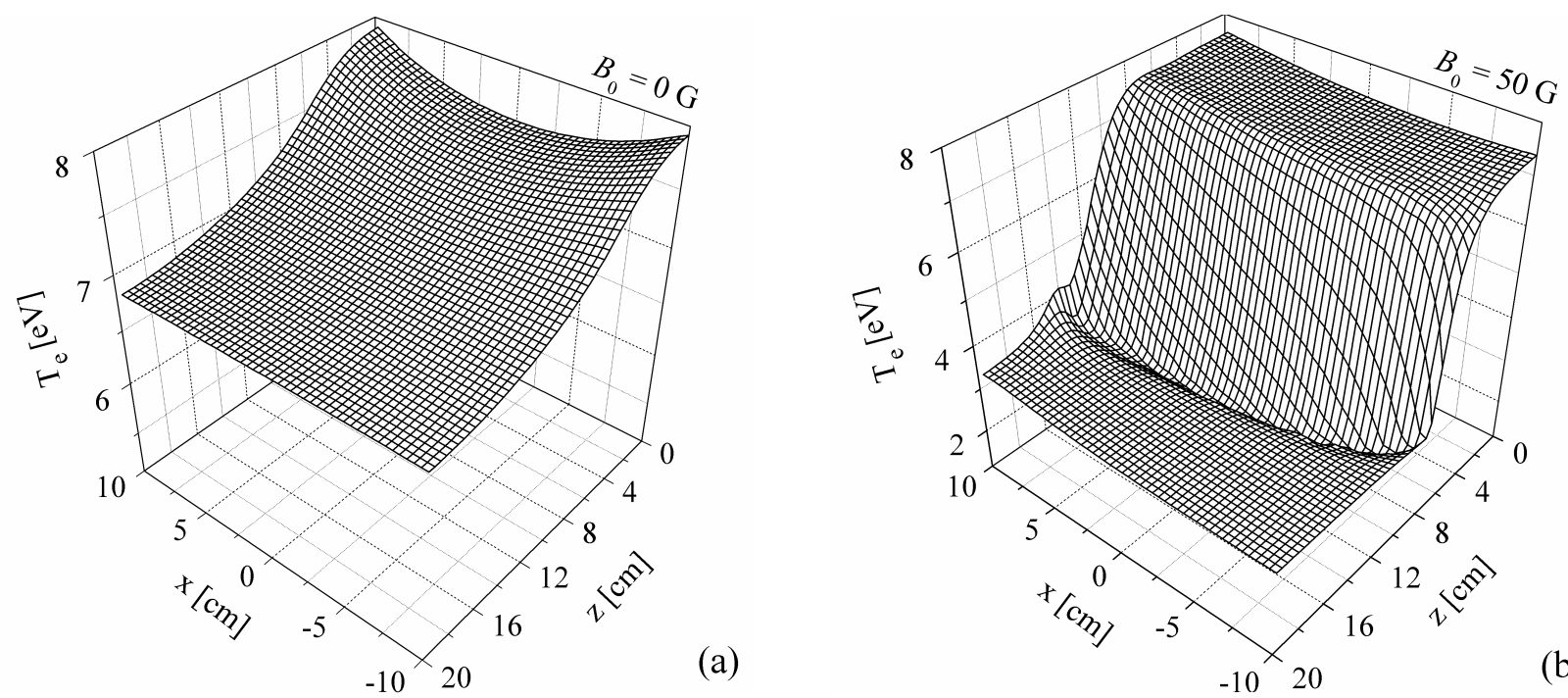

Figure 3. Spatial distribution of the electron temperature obtained within the $2 \mathrm{D}$ model for $B_{0}=0 \mathrm{G}$ and $B_{0}=50 \mathrm{G}(\mathrm{b})$.

The decrease of the electron density, both in the $z$ - and $x$ - directions for $B_{0}=0 \mathrm{G}$ (figure 4(a)) is due to an ordinary diffusion without magnetic field. E.g., the density profile in the $x$-direction at $z=0$ coincides with a cosine-profile, well-known $[28,29]$ for discharge description in Cartesian co-ordinates. Due to the comparatively low input power and, thus, comparatively low plasma density, sheath effects are not well pronounced. The picture of the 2D distribution of $n_{e}$ (figure 4(b)) for $B_{0} \neq 0$ is again - like that 
for $T_{e}$ - quite more complicated. The reason for a formation of a maximum of $n_{e}(z)$ in the region of the filter $(z=10 \mathrm{~cm})$ is the same as in the 1D model: thermal diffusion (due to the axial drop of $T_{e}$ ) acting together with diffusion. However, the maximum here is shifted from the center towards negative $x$-values. The $(\mathbf{E} \times \mathbf{B})$-drift in the $x$-direction due to the electric field in the $z$-direction (the fourth term in (11a)) is the reason for this. The formation of secondary maxima in the out-of-center region of the discharge is another modification of the spatial distribution of $n_{e}$ shown by the 2D-model. It is driven by the complicated spatial variation of the electron temperature (figure 3(b)) in the groove, in the filter region. Due to the minimum of $T_{e}$ in the groove, thermal diffusion fluxes in opposite directions arise, as described by the third term in (11c), which lead to the formation of the secondary maxima in the $n_{e}$ distribution.
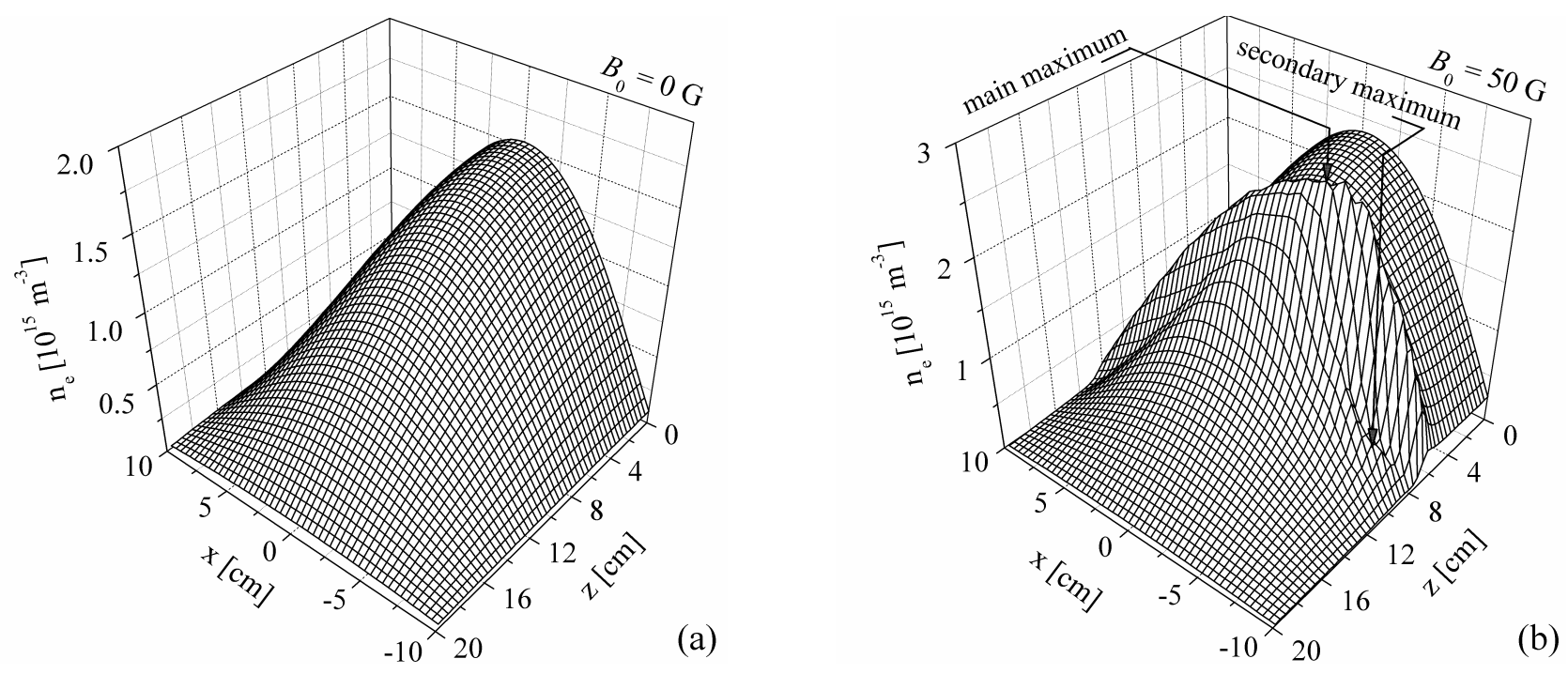

Figure 4. Spatial distribution of the electron concentration obtained within the $2 \mathrm{D}$ model for $B_{0}=0 \mathrm{G}$ (a) and $B_{0}=50 \mathrm{G}(\mathrm{b})$.
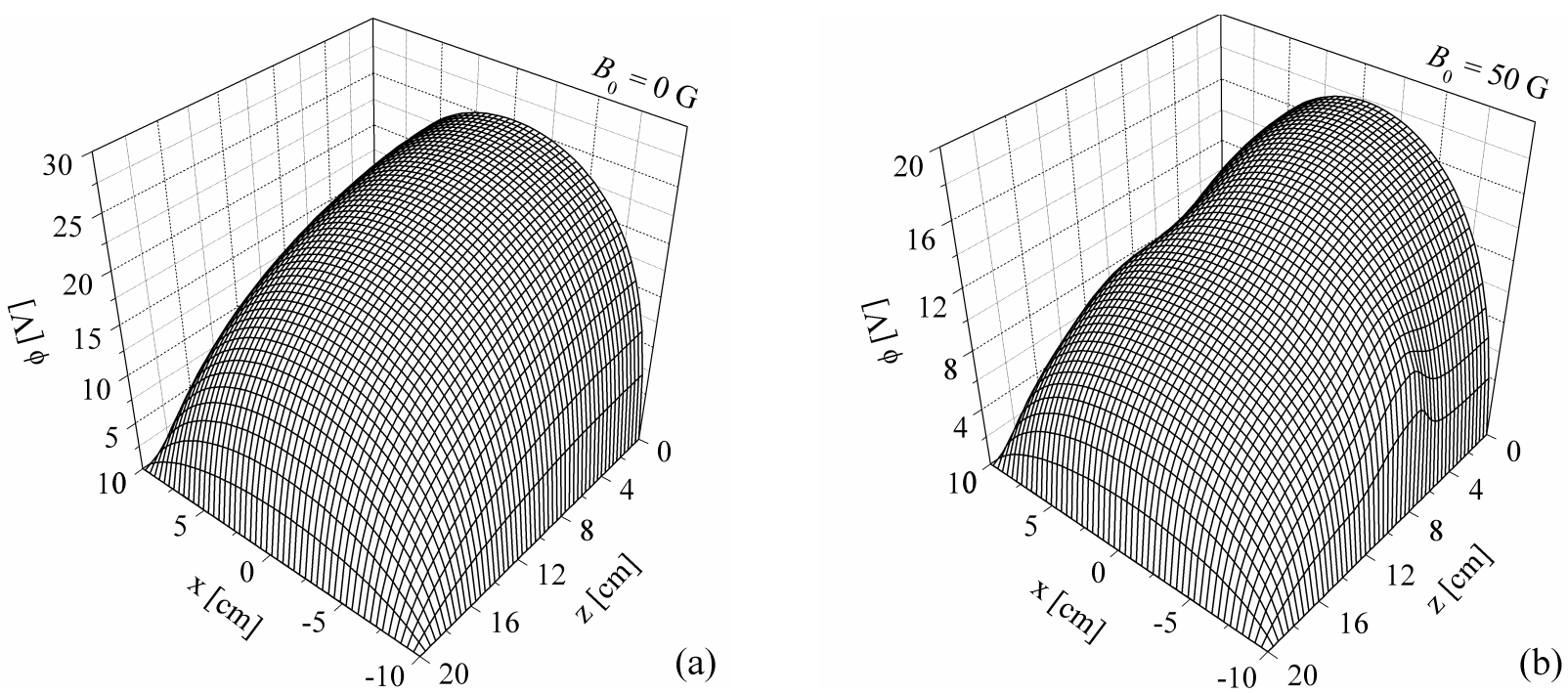

Figure 5. Spatial distribution of the potential of the dc electric field obtained within the 2D model for $B_{0}=0 \mathrm{G}$ (a) and $B_{0}=50 \mathrm{G}(\mathrm{b})$. 
With the values of the plasma parameters obtained within the 2D-model, the $\mathrm{H}_{2}^{+}$-ions appear to be the ions with the highest concentration. Similarly to the 1D-description, their spatial distribution is in the trends of the spatial distribution of the electron concentration. The same holds for the spatial distribution of the densities of the $\mathrm{H}^{+}$- and $\mathrm{H}_{3}^{+}$- ions.

Figure 5 shows the 2D-distribution of the potential of the dc electric field formed in discharges without and in an external magnetic field. With the zero value of the potential at the walls (21) and with the low values of the magnetic field, typical for the magnetic filters for electron cooling, the wall sheath is positive and, respectively, the potential in the plasma is positive. The peculiarities in the spatial distribution of the dc potential caused by the magnetic field (figure 5(b)) are in the region between the main and secondary maxima in the distribution of the charged particles (figures 4(b)). As it has been commented, the formation of the maxima of $n_{e}$ is due to the thermal diffusion of the electrons. On the other hand, $T_{i}=$ const., i.e., there is no thermal diffusion of ions and, as it has been mentioned, the ion concentration follows that of the electrons (figure 4(b)) due to tendency to quasi-neutrality. However, it appears that the quasi-neutrality is not complete and this leads to larger ion concentration compared to the concentration of the electrons and, respectively, to a local increase of the de potential.

The spatial distribution of the hydrogen atoms (figure 6) shows a decrease of their concentration $N_{a}$ in both ( $x$ - and $z$-) directions. The spatial distribution of the concentration $N_{m}$ of the hydrogen molecules is related to that of the atomic hydrogen according to (23). The density of the atomic hydrogen at $B_{0}=50 \mathrm{G}$ is slightly higher than that at $B_{0}=0 \mathrm{G}$ because the dissociation is more efficient when the electron density is higher [46]. With the constant value of the gas pressure, the density of the molecular hydrogen is slightly lower at $B_{0}=50 \mathrm{G}$ compared to the $\left(B_{0}=0 \mathrm{G}\right)$-case. The shape of the spatial distribution of the concentrations of the neutral species is almost the same without and with magnetic field.
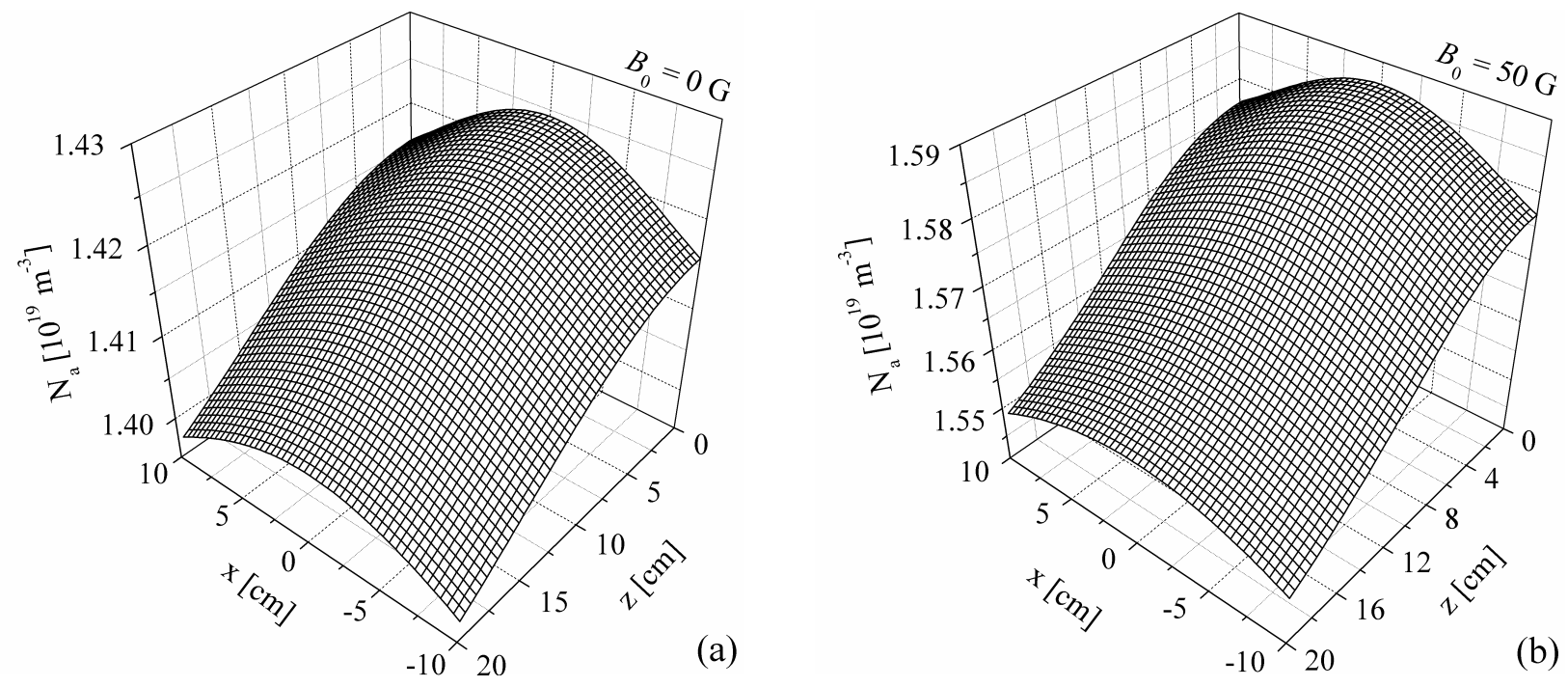

Figure 6. Spatial distribution of the atomic gas component obtained within the $2 \mathrm{D}$ model for $B_{0}=0 \mathrm{G}$ (a) and $B_{0}=50 \mathrm{G}(\mathrm{b})$.

Figure 7, which shows the dependence of the axial variations of the electron temperature and density on the magnetic field obtained at $x=0$ within the 2D-model, provides possibilities for a direct comparison with the corresponding results (figure 2) from the 1D-model. Besides the modifications in the distribution of the plasma parameters due to the $(\mathbf{E} \times \mathbf{B})$ - drift and the diamagnetic drift as well as to the thermal flux associated with the diamagnetic drift already discussed, the comparison shows that the 2Dmodel predicts higher electron temperatures and lower electron concentrations. Obviously, accounting for the second $(x)$ dimension brings significant losses due to the charged particle fluxes to the $\left(x= \pm L_{x}\right)$ - 
walls. The higher losses of charged particles require a higher electron temperature for the discharge maintenance. Since in both cases, of the 1D- and 2D-models, the applied power is the same, the electron density within the 2D model is lower, because the electron temperature there is higher. The comparison of figures 2 and 7 shows also that the 1D- and 2D-models predict different behaviour - with changing $B_{0}-$ of the plasma parameters in the region before the filter. Whereas within the 1D model the electron temperature at $z=0$ decreases with increasing $B_{0}$, the 2D-model predicts almost constant $T_{e}$ for varying $B_{0}$. The decrease of $T_{e}$ with growing $B_{0}$ obtained within the $1 \mathrm{D}$ model has been related to decreasing diffusion losses for higher $B_{0}$. Within the 2D model, the losses due to charged particle fluxes to the walls in the $x$-direction appear to be large not permitting lowering of $T_{e}$. The different $T_{e}\left(B_{0}\right)$ dependencies at $z=0$ result into different $n_{e}\left(B_{0}\right)$-dependencies, within the 1D- and 2D-models. Although in both cases $n_{e}(z=0)$ increases with growing $B_{0}$, in the 2D model this increase is weaker, due to the additional losses by the charged particle fluxes in the $x$-direction taken into account. By the same reason the peak of the electron density in the region of the filter is well pronounced within the 1Dmodel for $B_{0}$-values lower than those in the 2D-model.
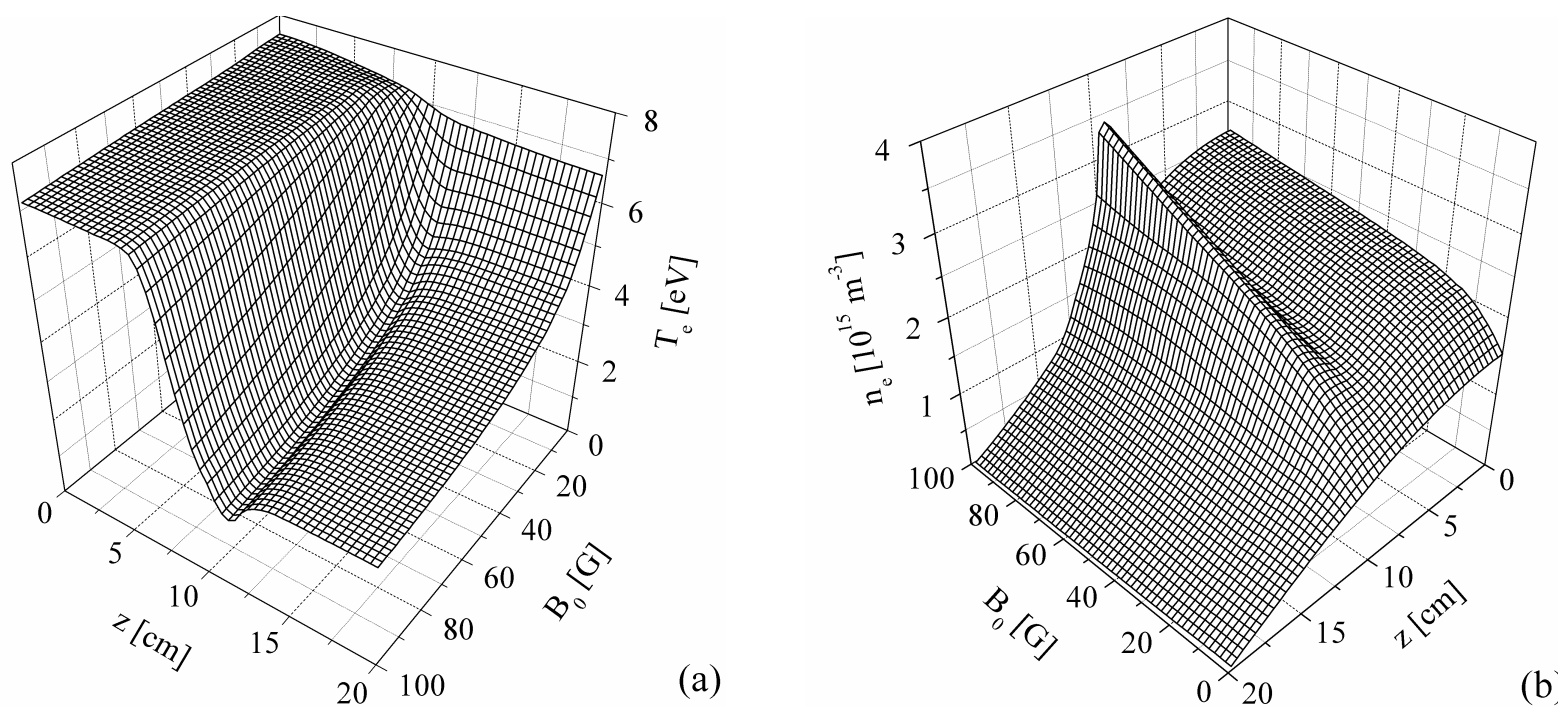

Figure 7. Changes of the axial distribution, for $x=0$, of the electron temperature (a) and density (b) for varying values of the magnetic field $B_{0}$ obtained from the $2 \mathrm{D}$ model.

Electron cooling is the purpose of using magnetic filters in the sources of negative hydrogen ions. However, keeping electron density as high as possible behind the filter is also desirable for the efficient production of negative ions. This means that the ratio $\left(\Delta n_{e} / \Delta T_{e}\right)$ of the density- and temperaturechanges across the filter region should be kept low. The results from the 2D-model show that after decreasing with the $B_{0}$-increase up to $20 \mathrm{G},\left(\Delta n_{e} / \Delta T_{e}\right)$ stays almost constant with a slight minimum at $B_{0}=60 \mathrm{G}$. Therefore, magnetic field values $B_{0} \geq 20 \mathrm{G}$ are proper ones regarding the requirement for small $\left(\Delta n_{e} / \Delta T_{e}\right)$-values.

The mechanism ensuring the plasma existence in the volume outside the power deposition region is illustrated in figure 8 where the axial changes of the contributions (at $x=0$ ) of the different terms $\left(P_{w}, P_{\text {coll }}\right.$ and $\left.\operatorname{div} \mathbf{Q}_{e}\right)$ to the electron energy balance (5) are plotted. Moreover, the $\operatorname{div} \mathbf{Q}_{\text {cond }}$ - and $\operatorname{div} \mathbf{Q}_{\text {conv }}$ - terms, where $\mathbf{Q}_{\text {cond }} \equiv \mathbf{q}_{e}$ and $\mathbf{Q}_{\text {conv }}=(5 / 2) n_{e} T_{e} \mathbf{v}_{e}$ (see, (7)), are presented separately in figure 8 , in order to stress on that the thermal flux is the flux responsible for the plasma maintenance outside the volume where the external power is applied. The extension of the region of the power deposition $P_{w}$ is over the distance of $z \leq 7 \mathrm{~cm}$. It compensates the local collisional losses $\left(P_{\text {coll }}\right)$ there and the losses due to the electron energy flux (both conductive and convective fluxes) which removes 
electron energy away from this region. With the $z$-decrease of the power input, the contribution of the conductive flux (the $\operatorname{div} \mathbf{q}_{e}$-term in (7)) changes in sign, i.e. it transfers from losses to a power input. The latter sustains the plasma in the expansion region compensating both the collisional losses there and the losses due to the convective flux. With magnetic field present (figure 8(b)), the electron thermal flux is reduced by the filter and the $\operatorname{div} \mathbf{Q}_{\text {cond }}$-term in the power input region is smaller than that for $B_{0}=0 \mathrm{G}$ (figure 8(a)). However, the collisional losses in the expanding plasma region are also smaller, due to the lower electron temperature and density there. The losses due to the convective flux are also reduced.
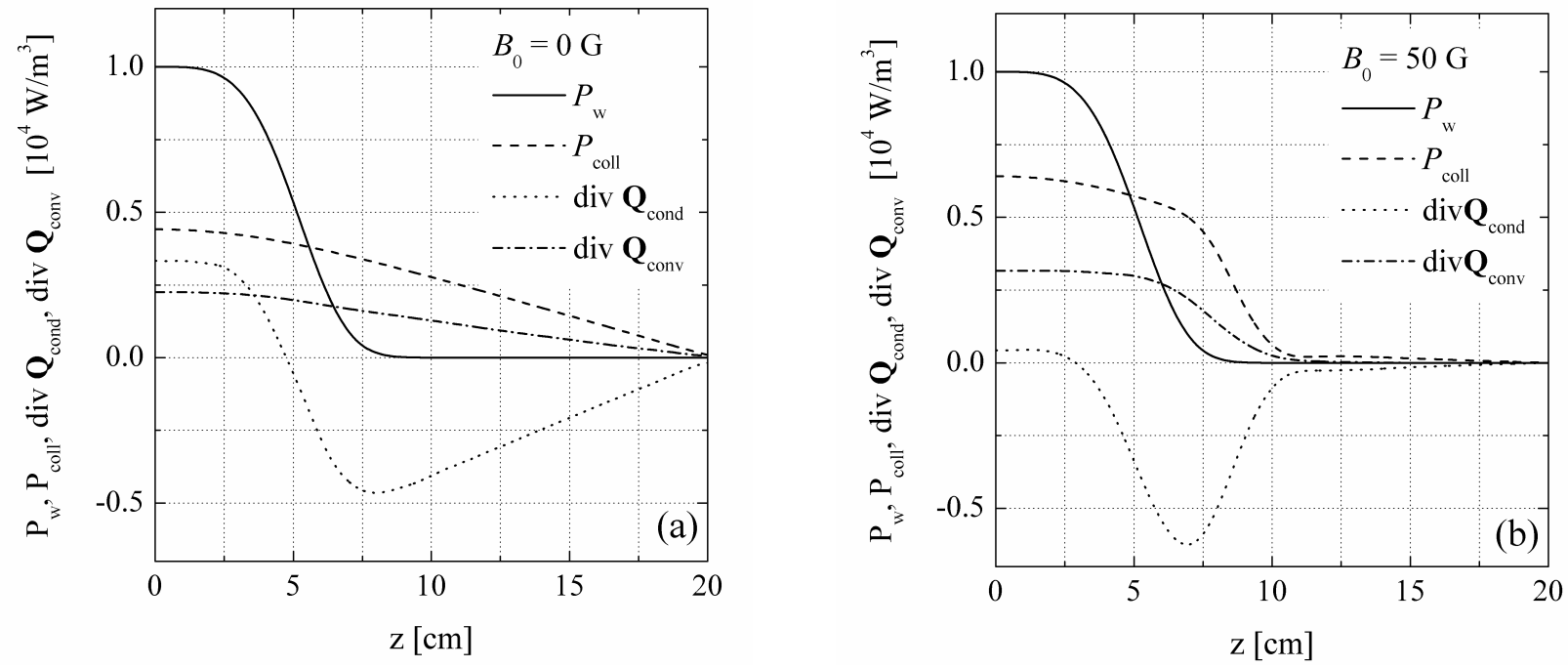

Figure 8. Axial changes, for $x=0$, of the different terms $\left(P_{w}, P_{\text {coll }}\right.$ and the contributions of the conductive and convective fluxes to $\operatorname{div} \mathbf{Q}_{e}$ ) in the electron energy balance (5) for $B_{0}=0 \mathrm{G}$ (a) and $B_{0}=50 \mathrm{G}(\mathrm{b})$; results from the 2D-model.
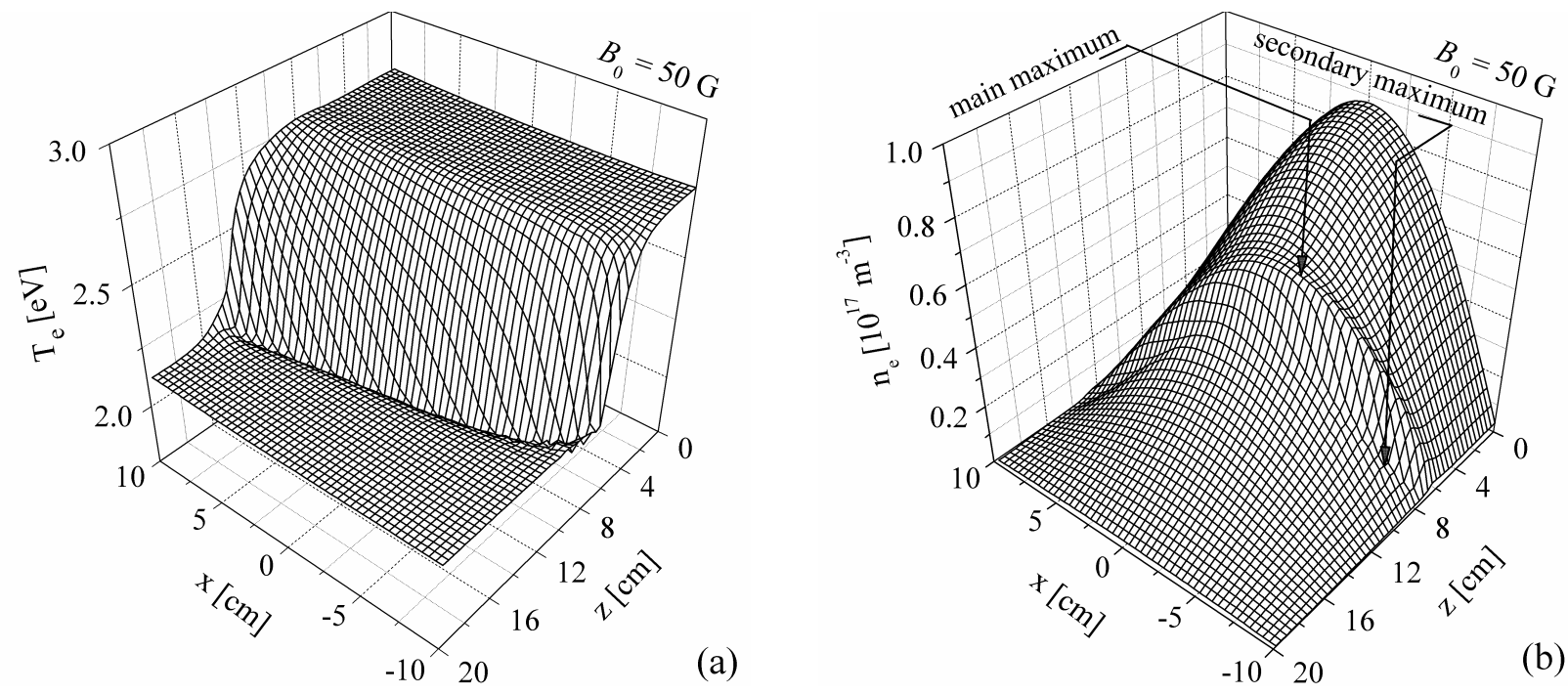

Figure 9. An argon discharge: spatial distribution of the electron temperature (a) and density (b) obtained within the 2D model.

An operation of the magnetic filter discussed in terms of electron-energy and charged-particle fluxes is based on general mechanisms of the behaviour of the gas discharges and, thus, it should not depend on the nature of the given gas. This is confirmed by the results shown in figure 9, for the space distribution of the electron temperature and density in an argon discharge, at $B_{0}=50 \mathrm{G}$. The charged-particle 
production is via direct and step ionization and the electron energy losses in collisions taken into account are through both inelastic and elastic collisions. The rate coefficients of the corresponding processes are obtained as in Ref. 47 by using the data for the cross sections and for the diffusion coefficient of the neutrals from [48-52]. The comparison of the results in figures 9(a) and 9(b) with the corresponding results (figures 3(b) and 4(b)) obtained in hydrogen discharges shows that all the effects discussed regarding the magnetic filter operation in hydrogen discharges appear also in argon discharges. The values of the electron temperature in the argon discharge are lower than those in the hydrogen discharge. However, this should be expected because of the lower losses in argon discharges. Respectively, since the applied power is kept the same, the plasma density in the argon discharge is higher. The relative drop of $T_{e}$ in the argon discharge is weaker compared to that in the hydrogen discharge and the maximum of the plasma density in the region of the filter is also less pronounced. As it has been discussed, the operation of the filter is mainly based on the electron fluxes. The ions are forced to follow the electrons, for keeping the quasineutrality. Probably, due to the higher mass of the argon ions, the ions in argon discharges do not permit too much freedom of the electron motion.

\subsection{D-model description}

Compared to the 2D-model of the magnetic filter operation, the 3D model presented here accounts for charged-particle and electron-energy fluxes in all, three $(x-, y$ - and $z$-), directions including the fluxes parallel to the magnetic field (in the $y$-direction). Hydrogen discharges are considered and the maximum value of the magnetic field of the filter is fixed to $B_{0}=50 \mathrm{G}$. The discussed results are for the spatial distribution of the electron temperature and density.
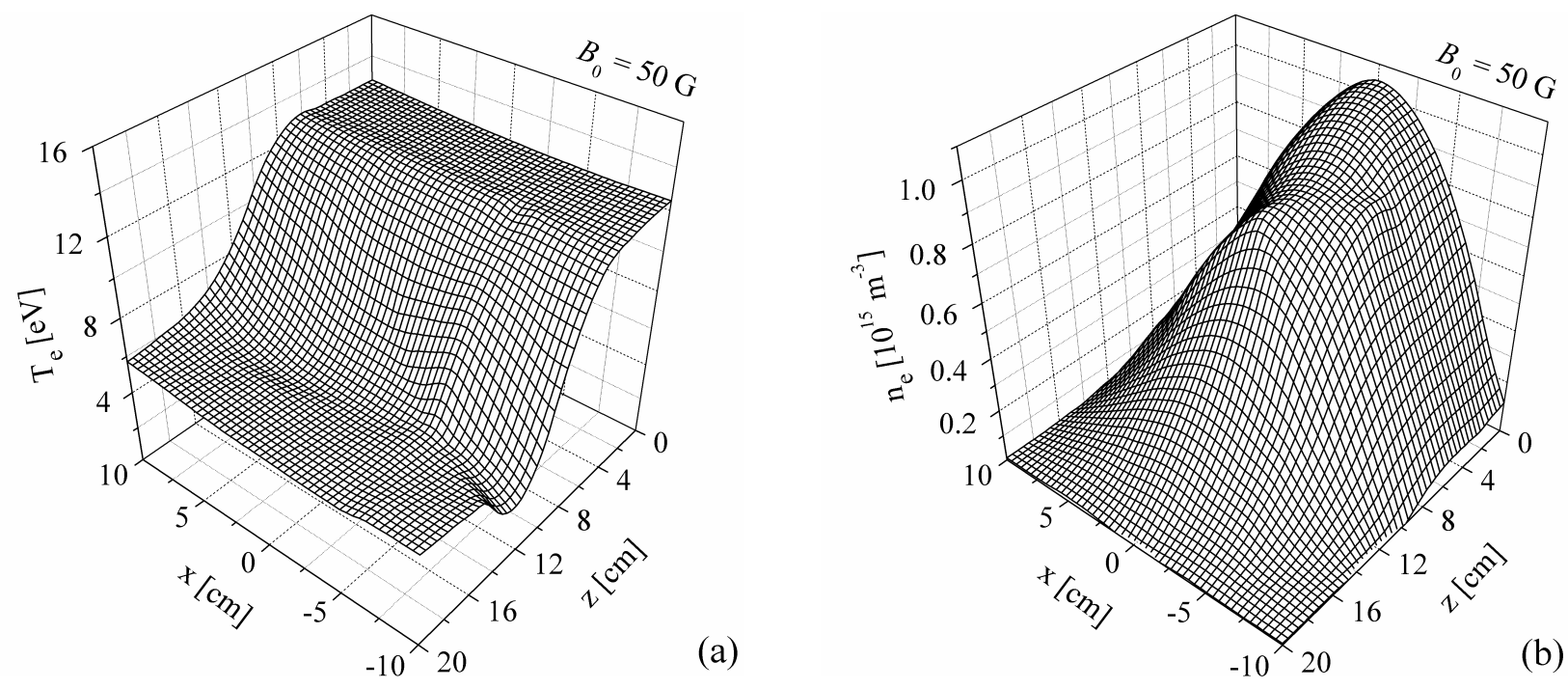

Figure 10. Results from the 3D-model: Spatial distribution of the electron temperature (a) and density (b) obtained in the $(x-z)$-plane, for $y=0$.

The general behaviour of the space distribution of $T_{e}$ and $n_{e}$ in the $(x-z)$-plane obtained within the 3D-model (figures 10(a) and 10(b)) is exactly the same as that predicted by the 2D model (figures 3(b) and 4(b)): axial (along the $z$-direction) drop of $T_{e}$ (figure 10(a)) with a formation of a groove in the filter region as well as formation of maxima of the electron concentration in the filter region. The obtained within the 3D model - higher values of the electron temperature and lower values of the electron concentration, compared to the results from the 2D-model, is in the trends of the comparison between the 1D- and 2D-models. Due to the third ( $y$-) direction taken into account in the 3D model and, respectively, due to charged-particle and electron-energy fluxes towards the $\left(y= \pm L_{y}\right)$-walls included, the total losses become higher requiring higher electron temperature for the discharge maintenance. Since the same power input is taken, the electron density is lower. 

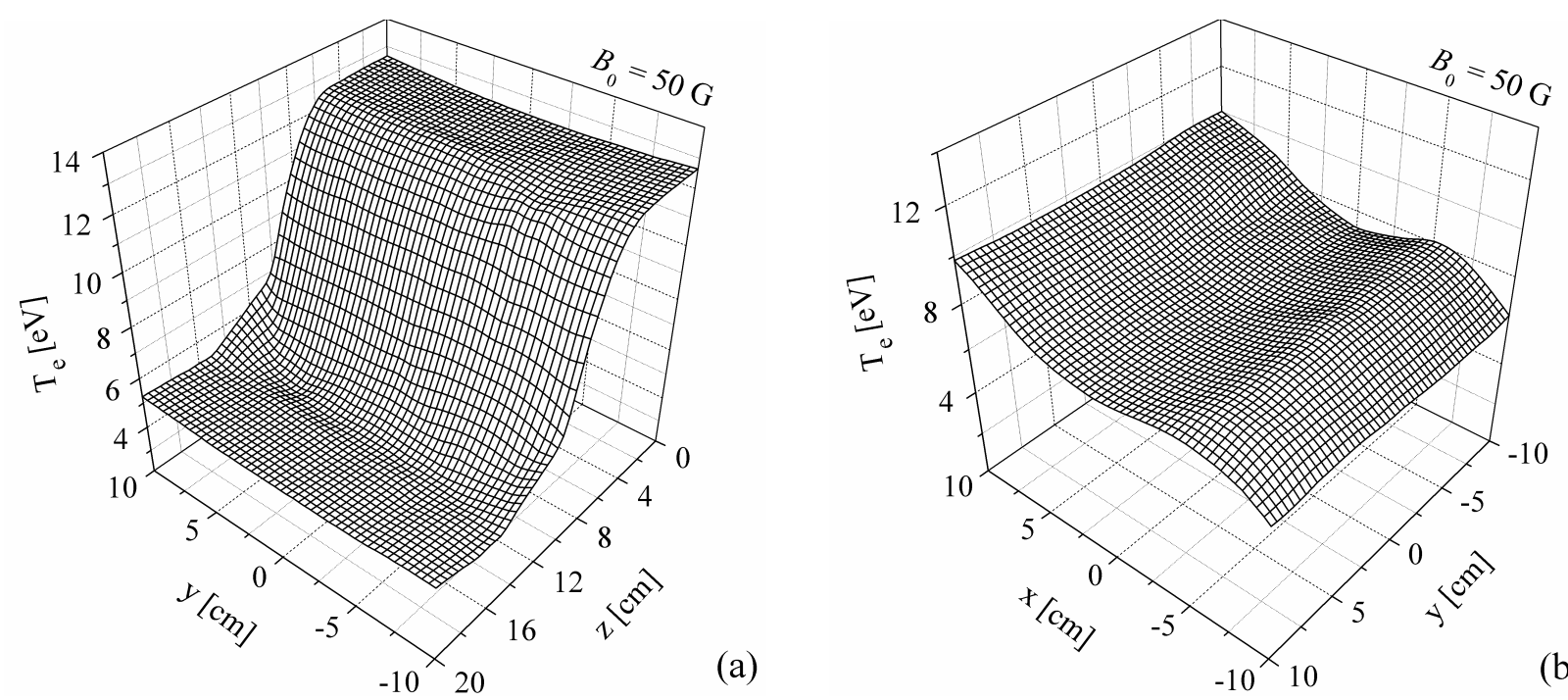

(b)

Figure 11. Results from the 3D-model: Spatial distribution of the electron temperature in the $(y-z)$-plane for $x=0$ in (a) and in the (x-y)-plane for $z \equiv z_{0}=10 \mathrm{~cm}$ in (b).

Figure 11 shows the spatial distribution of the electron temperature in the $(y-z)$-plane for $x=0$ and in the $(x-y)$-plane for $z=10 \mathrm{~cm}$, i.e. at the center of the filter where the groove is formed. In both planes, the electron temperature is almost constant in the $y$-direction. Therefore, although accounting for the chargedparticle and electron-energy fluxes along the magnetic field shifts the total distribution of the electron temperature towards higher values, it does not introduce new effects influencing the operation of the filter.

\section{Conclusions}

The study is on the mechanisms governing the operation of magnetic filters for electron cooling in lowpressure gas-discharges. Its analytical part presents a 3D-model description - based on the fluid plasma theory - of the spatial distribution of the plasma parameters of the discharges when localized inhomogeneous magnetic fields are externally applied. The filter operation is discussed based on numerical results obtained by complicating consecutively the treatment: (i) starting with an 1Ddescription of the variation of the plasma parameters along the discharge length (a direction perpendicular to the magnetic field), (ii) passing through a 2D description of the discharge structure in the middle plane of the discharge vessel, across the magnetic field, and (iii) ending with a 3D-model description of the whole discharge. With the weak magnetic field applied, only the electrons in the discharge are magnetized and, thus, the electron motion is that driving the changes in the spatial distribution of the plasma parameters caused by the magnetic field. Regarding the use of the magnetic filter for electron cooling in the sources of negative hydrogen ions, the discussion is based on hydrogen discharges.

The obtained results on the mechanisms of the filter operation can be summarized, as follows. Reduction of the thermal conductivity coefficient in a transverse magnetic field is responsible for the strong drop of the electron temperature in the region of the filter and behind it. The strong spatial variation of the electron temperature forwards importance of the thermal diffusion which together with the diffusion, both in a transverse magnetic field, forms a maximum of the electron concentration at the discharge center, in the region of the filter. Thermal fluxes related to the diamagnetic drift lead to inhomogeneity of the electron temperature in the second direction, which is also across the magnetic field, causing a formation of a minimum of the electron temperature in the region of the filter. The latter drives the thermal diffusion to form - together with the diffusion - a secondary maxima of the electron density in the out-of-the center region of the discharge. The $(\mathbf{E} \times \mathbf{B})$-drift shifts the main - central maximum of the plasma density from the discharge axis.

The 1D model provides the major result for the filter operation as an electron cooler, i.e. the drop of the electron temperature caused by the magnetic field. However, the $2 \mathrm{D}$ model describing the 
modification of the plasma parameters in the two directions, transverse to the applied magnetic field, is that providing the complete description of the mechanisms of its operation. The results obtained within the 3D model show that adding the third dimension - the direction parallel to the magnetic field - does not influence the pattern of the spatial distribution of the plasma parameters and, thus, it does not introduce new effects concerning the mechanism of the operation of the filter. However, due to the additional - compared to the 2D-model - losses taken into account, the electron temperature $T_{e}$ and the plasma density $n_{e}$ obtained in the 3D- and 2D- models are different in their values. The same concerns the product $n_{e} T_{e}$ which, in general, depends on both the losses and the power input. The higher losses due to the additional walls taken into account in the 3D-model, compared to the 2D-model, mean that higher electron temperature - as it is obtained - is needed for the discharge maintenance. The latter leads to a lower plasma density, when the applied power is the same, as here. Comparison of the results obtained with the 1D- and 2D- models shows the same. Since small changes of the electron temperature lead to large changes of the electron density (due to rate coefficients exponentially depending on $T_{e}$ ), the product $n_{e} T_{e}$ decreases with the increase of the dimensions.

The study is motivated by the use of the magnetic filters for cooling of the electrons in the sources of negative hydrogen ions. However, rather description of the general mechanisms of the operation of the filter than studying conditions for reaching the value of the electron temperature considered as the most proper one for effective production of negative ions is aimed at. These mechanisms, shown to be based on electron-energy and charged-particle fluxes, are common for discharges in different gases, as it has been demonstrated by presenting results not only for hydrogen discharges but also for discharges in an argon gas. Following the lines of looking for the general trends of plasma expansion through a magnetic filter, positioned outside the region of power input deposition, and for possibilities to compare the capabilities of the 1D- and 2D- model description with respect to the 3D-model description, the value of the applied power is kept the same. The value chosen is quite smaller than the usual one in the sources of negative hydrogen ion beams in order to have the initial set of equations for the 2D- and 3D-models valid also for the 1D-model. In the cases of the 2D- and 3D- models the Coulomb collision frequency is more than two orders of magnitude lower than the elastic electron-neutral collision frequency. Thus, even with the two orders of magnitude higher electron density when the input power is increased to the values usually used in the sources of negative ion beams, the elastic collision frequency would still stays higher than the frequency of the Coulomb collisions and the mechanisms of the filter operation will be the same, as described here. This is not the case when the description is within the 1D-model. Even with the low value of the power input taken here, the magnetic field could not exceed $50 \mathrm{G}$ due to the low losses in this case, which govern low electron temperature and, thus, high electron density.

The reliable operation of the numerical code developed in this study could be employed in future work on optimization of the magnetic filters in the sources of negative hydrogen ions regarding changes of the configuration of the magnetic field and of the gas-discharge conditions (gas pressure and applied power) as well as modifications of the discharge properties caused by accounting for the negative ions.

\section{Acknowledgments}

ASh greatly acknowledge the Alexander-von-Humboldt Foundation for the award of a Humboldt prize as well as Professor Dr. R. Wilhelm and Dr. E. Speth for their kind hospitality. KhT is also deeply obliged to the Alexander-von-Humboldt Foundation for the return fellowship. The work is within the EURATOMprogramme project FU06-CT-2003-00139, project no F-1315 of the National Science Fund in Bulgaria and project 2.3-BUL/1026323 supported by the Alexander-von-Humboldt Foundation.

\section{References}

[1] Brown G, ed 2004 The Physics and Technology of Ion Sources (Weinheim: Wiley)

[2] Loeb H W 2005 Plasma Phys. Control. Fusion 47, B565

[3] Speth E et al 2006 Nucl. Fusion 46, S220

[4] Holmes A J T 1992 Plasma Phys. Control. Fusion 34, 653

[5] Bacal M 1989 Nucl. Instrum. Methods Phys. Research B37/38, 28

[6] Holmes A J T 1982 Rev. Sci. Instrum. 53, 1517

[7] Holmes A J T 1982 Rev. Sci. Instrum. 53, 1523 
[8] Haas F A, Lea L M and Holmes A J T 1991 J. Phys. D: Appl. Phys. 24, 1541

[9] Holmes A J T, McAdams R, Proudfoot G, Cox S, Surrey E and King R 1994 Rev. Sci. Instrum. 65, 1153

[10] Shirai M, Ogasawara M, Koishimine T and Hatayama A 1996 Rev. Sci. Instrum. 67, 1085

[11] Holmes A J T 1996 Plasma Sources Sci. Technol. 5, 453

[12] Ohi K, Naitou H, Tauchi Y and Fukumasa O 2001 Phys. Plasmas 8, 23

[13] Kumar T A S, Mattoo S K and Jha R 2002 Phys. Plasmas 9, 2946

[14] Kraus W, McNeely P, Franzen P, Entscheva A, Bandyopadhyay M, Heinemann B, Riedl R, Speth E and Wilhelm R 2003 Fusion Eng. Design 66-68, 491

[15] Mizuno T, Kitade Y, Hatayama A, Sakurabayashi T, Imai N, Miroshita T and Inoue T 2004 Rev. Sci. Instrum. 75, 1760

[16] Leung K N, Hauck C A, Kunkel W B and Walther S R 1990 Rev. Sci. Instrum. 61, 1110

[17] Takeiri Y, Ando A, Kaneko O, Oka Y, Tsumori K, Akiyama R, Asano E, Kawamoto T and Kuroda T 1995 Rev. Sci. Instrum. 66, 2541

[18] Riz D and Paméla J 1998 Rev. Sci. Instrum. 69, 914

[19] Morishita T, Ogasawara M and Hatayama A 2000 Rev. Sci. Instrum. 71, 880

[20] Leung K N, Ehlers K M and Bacal M 1983 Rev. Sci. Instrum. 54, 56

[21] York R L, Stevens R R, Leung K N and Ehlers K W 1984 Rev. Sci. Instrum. 55, 681

[22] Inoue T, Araki M, Hanada M, Kurashima T, Matsuda S, Matsuda Y, Ohara Y, Okumura Y, Tanaka S and Watanabe K 1989 Nucl. Instrum. Methods Phys. Research B37/38, 111

[23] Leung K N et al 1990 Rev. Sci. Instrum. 61, 2378

[24] Fukumasa O, Naitou H and Sakiyama S 1993 J. Appl. Phys. 74, 848

[25] Morishita T, Miyamoto Y, Fujiwara Y, Hanada M, Kitagawa T, Kashiwagi M, Okumura Y and Watanabe K 2002 Rev. Sci. Instrum. 73, 1064

[26] Fukumasa O, Hosoda M and Naitou H 1991 Rev. Sci. Instrum. 63, 2696

[27] Naitou H, Ohi K and Fukumasa O 2000 Rev. Sci. Instrum. 71, 875

[28] Golant V E, Zhilinskiy A P and Sakharov I E 1977 Fundamentals of Plasma Physics (Moscow: Atomizdat), in Russian

[29] Lieberman M A and Lichtenberg A J 1994 Principles of Plasma Discharges and Material Processing (New York: Wiley)

[30] Boeuf J P and Pitchford L C 1995 Phys. Rev. E 51, 1376

[31] Hagelaar G J M, de Hoog F J and Kroesen G M W 2000 Phys. Rev. E 62, 1452

[32] Lymberopoulos D P and Economou D J 1995 J. Res. Natl. Inst. Stand. Technol. 100, 473

[33] Costin C, Marques L, Popa G and Gousset G 2005 Plasma Sources Sci. Technol. 14, 168

[34] Aliev Yu M, Schlüter H and Shivarova A 2000 Guided-Wave-Produced Plasmas (Berlin: Springer)

[35] Fukumasa O, Itatani R and Saeki S 1985 J. Phys. D: Appl. Phys. 18, 2433

[36] Chabert P, Rousseau A, Gousset G and Leprince P 1998 J. Appl. Phys. 84, 161

[37] St-Onge L and Moisan M 1994 Plasma Chem. Plasma Proc. 14, 87

[38] Koleva I, Paunska Ts, Schlüter H, Shivarova A and Tarnev Kh 2003 Plasma Sources Sci. Technol. 12, 597

[39] Janev R K, Langer W D, Evans K Jr and Post D F Jr 1987 Elementary Processes in HydrogenHelium Plasmas (Berlin: Springer)

[40] Neynaber R H and Trijillo S M 1968 Phys. Rev. 167, 63

[41] Mc Daniel E W and Mason E A 1973 The Mobility and Diffusion of Ions in Gases (New York: Wiley)

[42] Levitskii S M 1964 Book of Problems and Calculations in Physical Electronics (Kiev: Naukova Dumka) (in Russian)

[43] Smirnov B M 1978 Physics of Weakly Ionized Gas (Moscow: Nauka) (in Russian)

[44] Itakawa Yu 1974 At. Data Nucl. Data Tables 41, 1

[45] Weissman S and Mason E A 1962 J. Chem. Phys. 36, 794

[46] Paunska Ts, Schlüter H, Shivarova A and Tarnev Kh 2003 Plasma Sources Sci. Technol. 12, 608

[47] Kolev St, Schlüter H, Shivarova A and Tarnev Kh 2006 Plasma Sources Sci. Technol. 15, 744

[48] Ferreira C M and Loureiro J 1983 J. Phys. D: Appl. Phys. 16, 1611

[49] Böhle A and Kortshagen U 1994 Plasma Sources Sci. Technol. 3, 80 
[50] Vriens L and Smeets A H M 1980 Phys. Rev. A 22, 940

[51] Dothan F and Kagan Yu M 1981 J. Phys. D: Appl. Phys. 14, 183

[52] Ferreira C M, Loureiro J and Ricard A 1985 J. Appl. Phys. 57, 82 